\title{
\begin{tabular}{l|l} 
Mibraries & DSpace@MIT
\end{tabular}
}

\author{
MIT Open Access Articles
}

An Electronic Force Sensor for Medical Jet Injection

The MIT Faculty has made this article openly available. Please share how this access benefits you. Your story matters.

As Published: $10.1115 / 1.4043196$

Publisher: ASME International

Persistent URL: https://hdl.handle.net/1721.1/135171

Version: Final published version: final published article, as it appeared in a journal, conference proceedings, or other formally published context

Terms of Use: Article is made available in accordance with the publisher's policy and may be subject to US copyright law. Please refer to the publisher's site for terms of use. 
Nickolas P. Demas ${ }^{1}$

Biolnstrumentation Laboratory, Department of Mechanical Engineering, Massachusetts Institute of Technology, Cambridge, MA 02139 e-mail: demas@alum.mit.edu

Ian W. Hunter George N. Hatsopoulos Professor in Thermodynamics, Biolnstrumentation Laboratory, Department of Mechanical Engineering, Massachusetts Institute of Technology, Cambridge, MA 02139 e-mail: ihunter@mit.edu

\section{An Electronic Force Sensor for Medical Jet Injection}

In medical jet injection, a narrow fluid drug stream is propelled at high velocity into skin without a needle. Previous studies have shown that the volume delivered is highly dependent on a number of factors. This paper details the development of an electronic force sensor for medical jet injection and shows that the normal contact force exerted on the tissue by the nozzle is an additional factor affecting volume delivered. Using this sensor, we measure the forces at the nozzle tip in the normal direction with a sensitivity of $18 \mu N$, calibrated over a range from $1 \mathrm{~N}$ to $8 \mathrm{~N}$ with a mean absolute error of $8 \mathrm{mN}$, and a maximum overload of $300 \mathrm{~N}$. We further measure forces at the nozzle tip in the lateral direction with a sensitivity of $8 \mu \mathrm{N}$, calibrated over a range from $0.1 \mathrm{~N}$ to $7 \mathrm{~N}$, with a mean absolute error of $101 \mathrm{mN}$ for lateral contact force magnitude and $1.60 \mathrm{deg}$ for lateral contact force direction. Experimental validation confirms that the force sensor does not adversely affect the accuracy and precision of ejected volume from the jet injector. We use this setup to examine the effect of normal contact force on volume delivered into postmortem porcine tissue. Experimental results demonstrate that volume delivered with normal contact force between $4 \mathrm{~N}$ and $8 \mathrm{~N}$ is significantly more accurate and precise compared to volume delivered with normal contact force between $0 \mathrm{~N}$ and $3.9 \mathrm{~N}$.

[DOI: $10.1115 / 1.4043196]$

\section{Introduction}

Medical jet injection is a drug delivery method where a small diameter $(50-250 \mu \mathrm{m})$, high-velocity $(100-350 \mathrm{~m} / \mathrm{s})$ jet of fluid drug penetrates tissue without a hypodermic needle [1]. Jet injectors with single-use nozzles do not suffer from crosscontamination issues present with mass-campaign multi-use nozzle jet injectors [2] and have emerged as a viable method for delivering insulin, anesthetics, and other transdermal treatments [3-5]. Jet injectors offer a potential reduction in pain due to the small liquid jet diameter and eliminate many issues associated with needle fear and needlestick injuries.

The International Organization for Standardization (ISO) has determined performance specifications for the accuracy of needle-based injection systems for medical use [6]. For jet injection to compete with traditional needle delivery, the accuracy of delivered volume must be matched. ISO 11608-1:2014 stipulates that needle-based devices must meet a tolerance interval such that they deliver at least $93 \%$ of all doses within the upper and lower specification limits. For single-dose (B2) devices, the upper and lower specification limits are specified as an absolute error of $10 \mu \mathrm{L}$ if the dose is below $200 \mu \mathrm{L}$ and a relative error of $5 \%$ if the dose is above $200 \mu \mathrm{L}$. Studies have shown that commercial needle-based injectors meet these requirements [7].

The process by which a jet injector's fluid stream penetrates tissue is much more complicated than needle-based hypodermic fluid delivery. Studies have shown that the volume delivered is highly dependent on the velocity of the fluid stream, nozzle diameter, nozzle shape, and stand-off distance between the nozzle and the tissue [1,8-13]. This knowledge has spurred efforts to develop strategies to control these parameters. For example, new injectors allow for the prescription and control of the jet velocity during the injection using closed-loop control $[1,14,15]$. This velocity profile in closed-loop controlled jet injectors can be tuned for particular

\footnotetext{
${ }^{1}$ Corresponding author.

Manuscript received July 11, 2018; final manuscript received February 28, 2019; published online April 11, 2019. Assoc. Editor: Michael Eggen.
}

individuals, injection sites, or medications for optimized delivery of treatment.

Even with careful control of jet velocity, nozzle diameter, nozzle shape, and stand-off distance between the nozzle and tissue, wet injections are still a problem with jet injectors. Wet injections occur when a portion of the liquid drug remains on the skin surface after the injection. The potential of a sizable and variable fraction of the prescribed dose failing to penetrate the tissue is problematic. We hypothesized that the magnitude of the normal contact force exerted on the tissue by the nozzle would influence the severity of wet injections (where severity is measured by the volume of liquid remaining on the tissue surface) which would, in turn, affect the volume delivered to the tissue.

In order to test this hypothesis, a force sensor capable of characterizing normal and lateral forces applied to the tip of the nozzle was needed. Figure 1 shows a range of possible contact force configurations that needed to be distinguished. We developed a novel, miniaturized, multi-axis electronic force sensor [16,17], which allows for the measurement of contact forces on a jet injector nozzle. These measurements can be displayed to guide a user to apply a predetermined normal contact force and minimize lateral forces. The measurement system can furthermore capture the state of all contact forces at the moment of injection. Following calibration and validation of the force sensor, we performed injections into postmortem porcine tissue and experimentally varied the normal contact force while minimizing lateral forces. Results indicate that elevated normal contact force levels increase accuracy and precision of drug volume delivered to the tissue.

\section{Methods}

2.1 Constraints. We determined that a user could maintain a force between $0 \mathrm{~N}$ and $10 \mathrm{~N}$ within an approximate range of $\pm 0.5 \mathrm{~N}$ [16] using the pistol-grip jet injector previously described in the literature [1] when the user was presented with an interface that displayed real-time force measurements. Additionally, normal contact forces above $10 \mathrm{~N}$ can cause discomfort. We therefore used $10 \mathrm{~N}$ as the upper force limit at which the device would be applied to skin. We further required that the sensitivity and standard deviation of the force measurement be less than $0.5 \mathrm{~N}$. For 


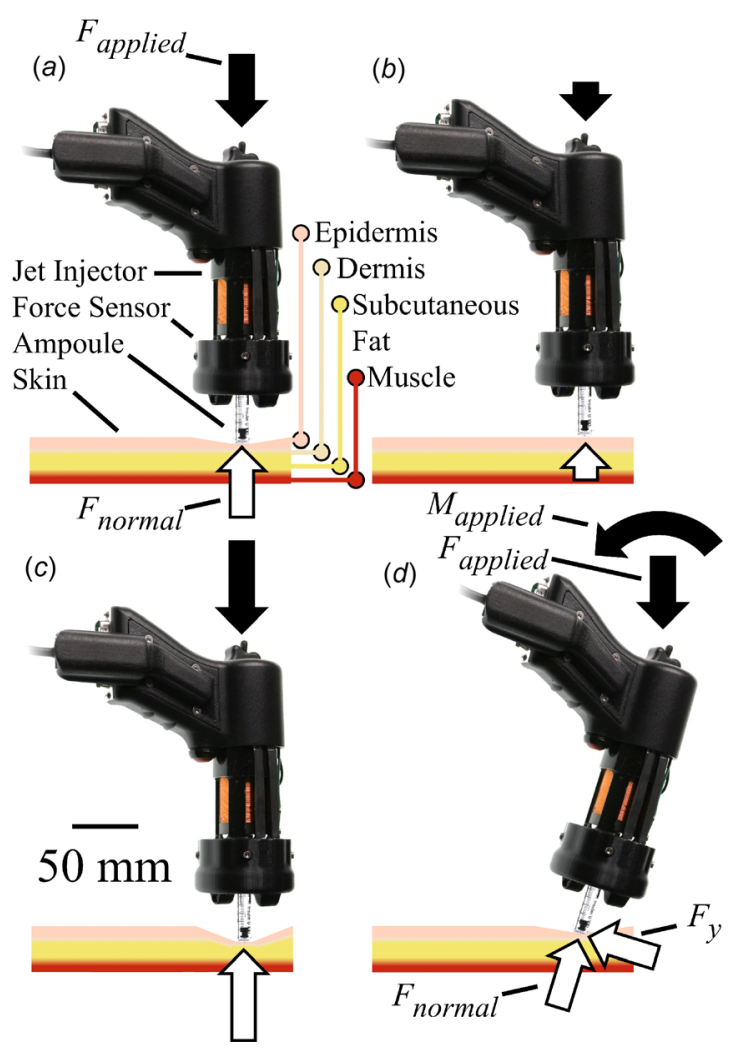

Fig. 1 Possible contact force configurations showing the skin, ampoule, force sensor, and jet injector. (a) shows a desired loading condition where normal force is applied at a desired level and lateral forces $\left(F_{X}\right.$ and $\left.F_{y}\right)$ are minimized. Also shown are nonideal loading conditions including $(b)$ insufficient normal force, $(c)$ excessive normal force, and (d) excessive lateral force (in this case along the $y$-axis).

clarity, sensitivity is the minimum possible increment that the sensor is able to detect, whereas standard deviation gives a measure of how measured noise affects precision. We also determined that the sensor needed to repeatably withstand an overload normal force of $300 \mathrm{~N}$, which was $150 \%$ of the maximum force that could be exerted by the pre-existing actuator [1].

We required that the force sensor have a bandwidth of at least $5 \mathrm{kHz}$, which was far above the fundamental resonance of the actuator at $400 \mathrm{~Hz}$ [1] and did not pose a risk of destabilizing the closed-loop actuator control system.

While modern sensing methods have made the required displacement very small, all methods of measuring forces require some displacement of a sensing element. We set the maximum volume error induced by the force sensor at $0.25 \mu \mathrm{L}$, which was over five times less than the standard deviation of $100 \mu \mathrm{L}$ injections by the jet injection device without a force sensor installed $[1,16]$ and forty times less than the strictest ISO specification [6]. Given the ampoule (Injex U-100) inner diameter of $3.5 \mathrm{~mm}$, we set the maximum allowable axial displacement at no more than $25 \mu \mathrm{m}$.

Finally, the sensor needed to meet a number of qualitative functional requirements. The sensor needed to be shielded from spurious loads, noise, and electrical interference caused by the jet injector. The sensor needed to reliably measure forces regardless of injector orientation or ambient temperature. Given that we were installing this sensor on a pre-existing jet injector [1], the force sensor needed to be readily integrated with this injector, which imposed design restrictions on size and geometry. Finally, the sensor also needed to be actuator-agnostic such that if the actuator was redesigned, the force sensor would not also require a complete redesign as well [16].

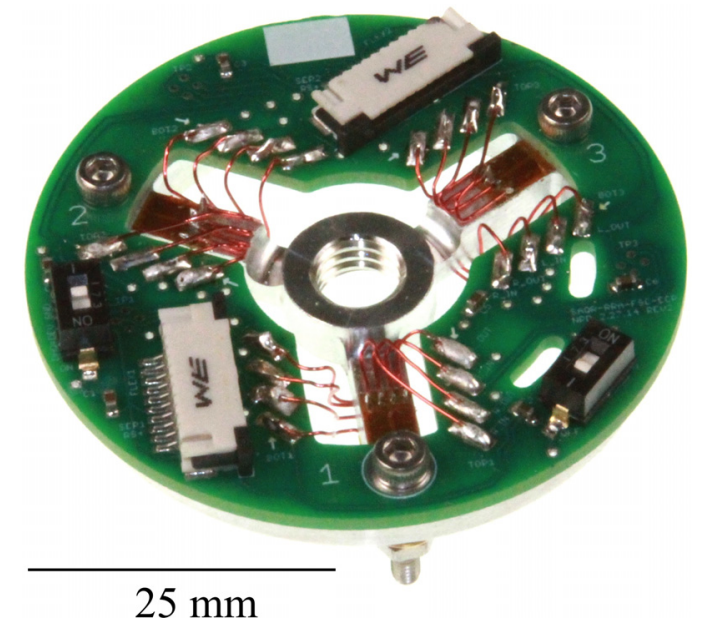

Fig. 2 Assembled force sensor alone without adapter, ampoule, or housing with the strain relief PCB mounted on top

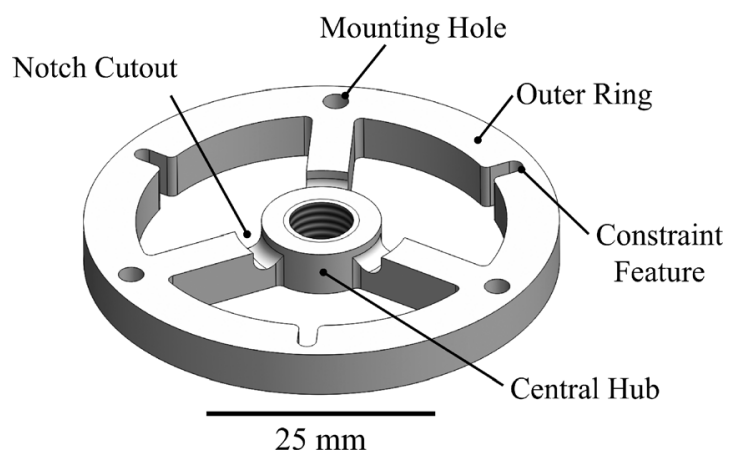

Fig. 3 Flexural beam mechanical design shown without strain gauges or strain relief PCB. Note the notch cutout at the inside edge of the flexures.

2.2 Design. The sensor we developed is shown in Fig. 2. This sensor consists of three flexural beams machined in 7075-T6 aluminum alloy, each approximately $13.2 \mathrm{~mm} \times 4.4 \mathrm{~mm} \times 5 \mathrm{~mm}$, connecting a central hub to an outer ring (Fig. 3). An M8 thread is machined in the central hub to interface with the ampoule. The outer ring mounts to a custom adapter made from 6061-T6 aluminum alloy, which connects the sensor to the actuator housing. This adapter can be modified to mount the force sensor on a different actuator if the actuator is redesigned in the future. The flexural beams have a notch machined near the central hub where the height of the flexure is significantly reduced. In simulations [16], flexural beams with this particular notch had measured strain distributions that were similar to guided flexural beams with approximately $10 \%$ larger length, allowing for the sensor size to be reduced while maintaining comparable surface strains. We found that a height at the notch of $2.5 \mathrm{~mm}$, cut with a $3.00 \mathrm{~mm}$ ball end mill to reduce stress concentrations, delivered desired performance. While a deeper notch would have allowed the beam length to be further decreased, elevated stresses experienced in the structure would lead to premature failure from cyclical loading in 7075-T6 aluminum as indicated by the literature [18] and finite element analysis.

Each flexural beam utilized four strain gauges in a full Wheatstone bridge configuration. This configuration was sensitive to vertical bending while rejecting lateral bending, torsion, axial loading, and thermal effects on each individual beam. Mounting locations on the top and bottom of the flexural beams near the fixed end were degreased (Maintex Citrus Degreaser), lightly abraded with 400 grit sandpaper, and rinsed with distilled water in preparation for bonding strain gauges. Six dual-bridge strain 
gauges (Omega SGD-3/350-DY41) were mounted to the specified locations using cyanoacrylate (Loctite 498). Attention was paid to alignment, but no procedures were used other than visual inspection to ensure correct placement.

We designed a custom strain relief printed circuit board (PCB). This PCB provided strain relief decoupling to eliminate any sensitivity to loads exerted on the sensor cable. We used 28 AWG copper magnet wire (TEMCo) to connect the strain gauges to the strain relief PCB as seen in Fig. 4. Two ten-pin flat flex cables (Würth Elektronik) allowed for power input and analog measurement output for all three full-bridge circuits. Connections were made through a series of cables to an NI-cRIO-9024 through an NI-9237 module (National Instruments). The NI-9237 module has 24-bit resolution on each measurement channel and an input range of $\pm 25 \mathrm{mV} / \mathrm{V}$. Additionally, we record the normal and lateral forces in the $10 \mathrm{~ms}$ prior to a trigger. Given the sampling rate of $50 \mathrm{~K} \mathrm{~S} / \mathrm{s} / \mathrm{ch}$ for the NI-9237 module, we record 500 normal and lateral force measurements over this time. Given an excitation voltage of $10.0 \mathrm{~V}$ and a sample length of 500 points, we calculate the $F_{\text {normal }}$ sensitivity to be $18 \mu \mathrm{N}$ given Eq. (2). Likewise, we calculated the $F_{x}$ and $F_{y}$ sensitivity to be $8 \mu \mathrm{N}$ given Eqs. (3) and (4). This sensitivity exceeds the design constraint of $\pm 0.5 \mathrm{~N}$ by a several orders of magnitude.

The sensor was fabricated and assembled in-house. The sensor was mounted on the pre-existing jet injector [1] (Figs. 5 and 6).

To measure the normal and lateral contact forces applied to the nozzle, we developed a model that took in measured voltages from the full-bridge circuits and calibration parameters. We applied an excitation voltage of $10.0 \mathrm{~V}$ across the Wheatstone bridge. Normal contact force was determined using the measured voltage $V_{n}$ along with the calibration coefficient $C_{n}$ and offset voltage $Z_{n}$ for each flexural beam $n$. The vertical load supported by each flexural beam is represented in Eq. (1), which indicates the load $F_{n}$ supported by the $n$th flexural beam.

$$
F_{n}=C_{n}\left(V_{n}-Z_{n}\right)
$$

The normal contact force applied to the nozzle was therefore the sum of the loads supported by each flexural beam as in the following equation:

$$
F_{\text {normal }}=F_{1}+F_{2}+F_{3}
$$

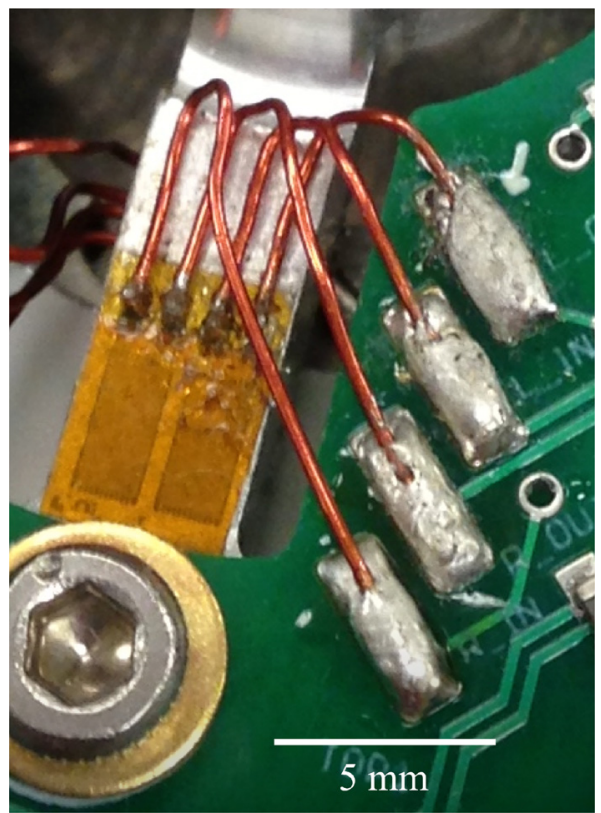

Fig. 4 Detail of electrical connections made with magnet wire between a dual-bridge strain gauge and the strain relief PCB

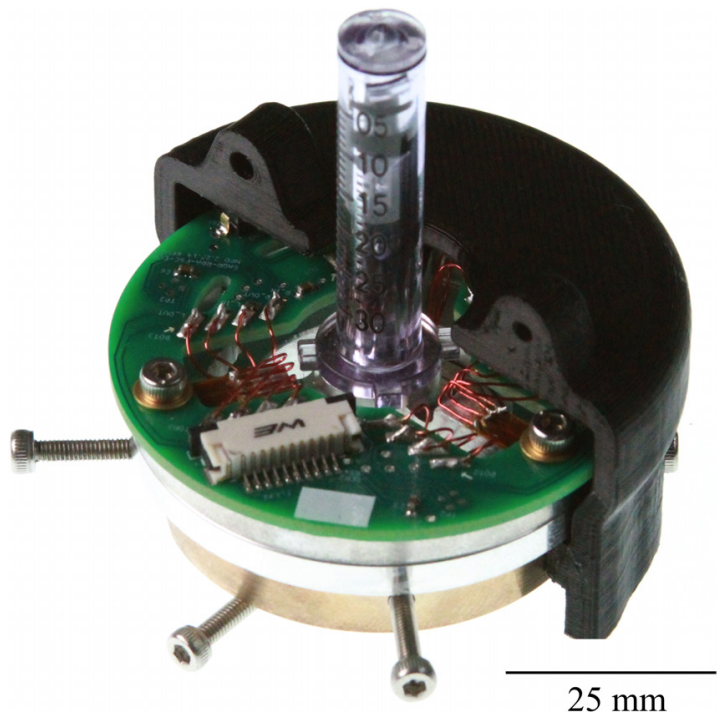

Fig. 5 Cutaway view of force sensor mounted on adapter with ampoule and housing

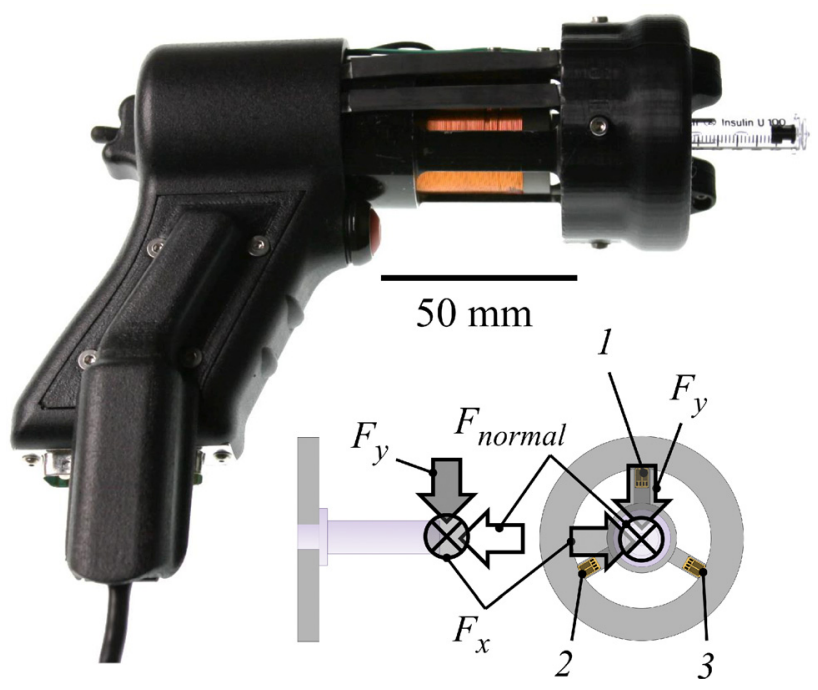

Fig. 6 Force sensor mounted on the pre-existing jet injector at top [1]. Illustrations of the three-flexure sensor (with the location of each flexural beam $n$ noted) and the alignment with $F_{\text {normal, }}, F_{x}$, and $F_{y}$ with $\Theta$ equal to zero are shown at bottom with a left side view (left bottom) and a front view (right bottom).

To determine the lateral force, a number of assumptions were made for the model including:

- The strain gauge configuration made the instrument only sensitive to vertical bending of the flexures.

- Vertical bending is linearly related to the magnitude of the applied lateral force on the nozzle.

- The degree of vertical bending (which is measured) versus torsion (which is rejected) is linearly related to the angle of the applied lateral force on the nozzle.

Equations for unscaled representations of the lateral force, $X$ and $Y$, in both lateral directions were formulated based on these assumptions ((in the following equations)):

$$
\begin{gathered}
Y=-F_{1}+\frac{F_{2}}{2}+\frac{F_{3}}{2} \\
X=-F_{2} \frac{\sqrt{3}}{2}+F_{3} \frac{\sqrt{3}}{2}
\end{gathered}
$$


Rotational correction of the measured lateral force representations (determined by geometry) aligned the user's expected axes with the device's axes. Skew correction (determined through experimentation) corrected for minor deviations from the expected behavior (see Appendix). Final scaling was accomplished with two lateral linear calibration coefficients (in the following equations):

$$
\begin{aligned}
& F_{x}=L_{x} X_{\text {skew }} \\
& F_{y}=L_{y} Y_{\text {skew }}
\end{aligned}
$$

2.3 Calibration and Validation. We designed a calibration jig (Fig. 7) to impart only normal contact force on the nozzle of the jet injector. This force caused equally distributed vertical bending among the three flexural beams. We suspended calibrated masses from the jig and recorded the resultant voltage. An $F_{\text {normal }}$ range of $1-8 \mathrm{~N}$ was applied during calibration. We fit a linear regression to the data using least squares (Fig. 8). Similar relationships were found for the other flexural beams and the parameters $C_{1}, C_{2}$, and $C_{3}$ were determined. Once these parameters had been inputted into the model, an $F_{\text {normal }}$ range of $1-8 \mathrm{~N}$ was again applied (Fig. 9). The measured $F_{\text {normal }}$ mean absolute error from the applied force is $8 \mathrm{mN}$ with a maximum absolute error of $13 \mathrm{mN}$ and no systematic trends. The average standard deviation of each individual measurement is $127 \mathrm{mN}$. These characteristics meet the specified constraints in Sec. 2.1.

We designed another calibration jig (Fig. 10) to impart only lateral contact force on the nozzle of the jet injector. A lateral calibrator plate consisted of an aluminum disk with regularly spaced pins. Monofilament line, which was anchored to the nozzle, was guided by these pins to precisely impart a particular lateral contact force direction. We located a chemistry ring stand fitted with a

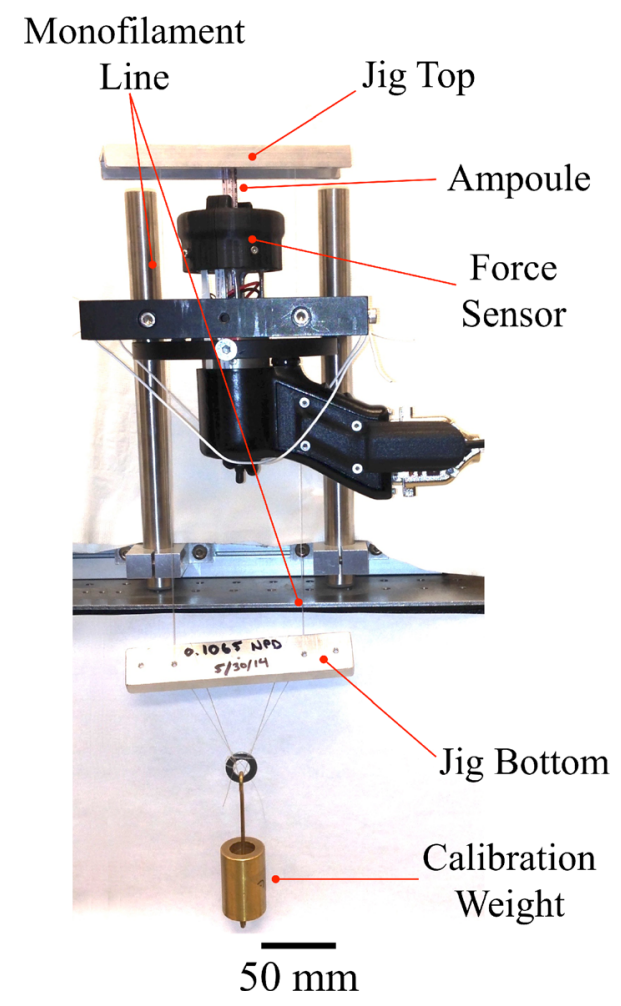

Fig. 7 The normal contact force calibration jig being used to calibrate normal contact force. The jig top rests on the nozzle of an upright ampoule mounted in the injector. Monofilament line connects the calibration weight to the jig top, applying a normal contact force.

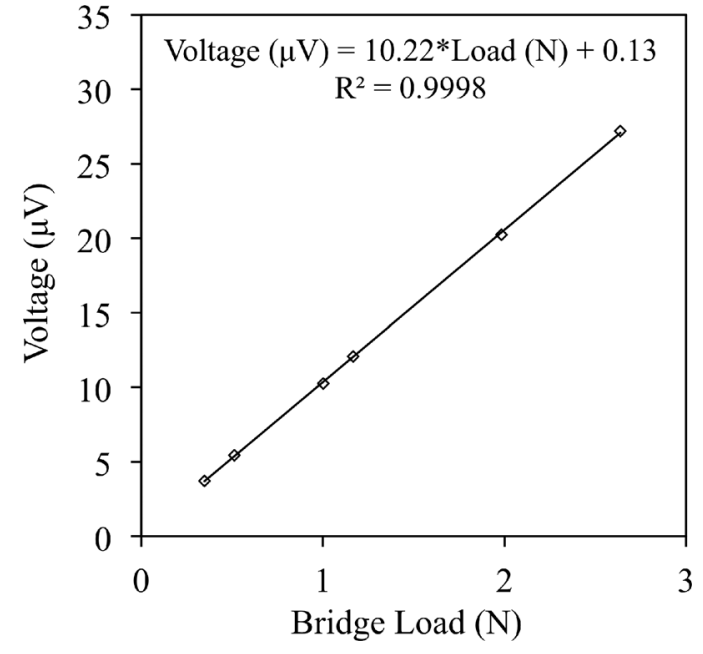

Fig. 8 Measured bridge voltage versus flexural beam load. The error bars, which indicate one standard deviation above and below the mean, are obscured behind the data points. $n=100$ for each discrete force level. The reported equation (and $R^{2}$ value) is for the line shown, fit using least squares.

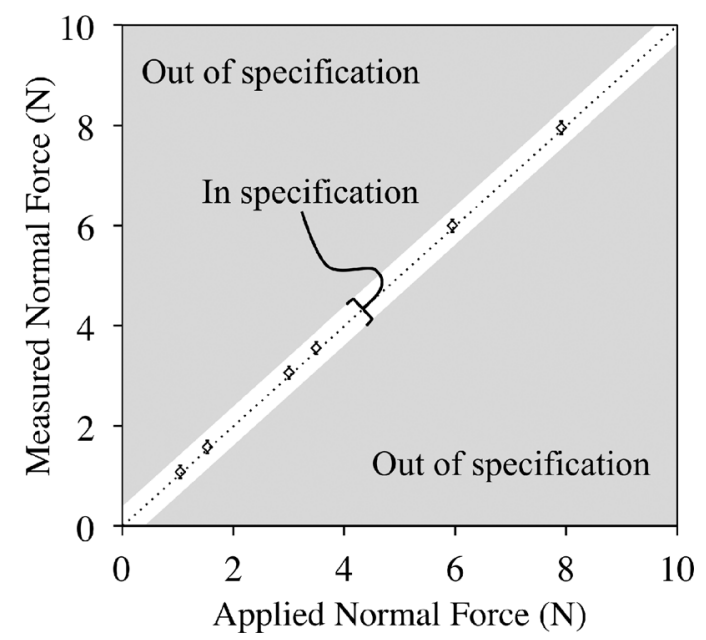

Fig. 9 Measured normal contact force versus applied normal contact force. Dotted line indicates perfect agreement between applied and measured values. Shaded regions show where $\pm 0.5 \mathrm{~N}$ is exceeded. The unshaded region conforms to the specification. All measurements fall in the unshaded region and conform to the specification. The error bars indicate one standard deviation above and below the mean. $n=100$ for each discrete force level.

pulley so that the monofilament line would lightly rest against the appropriate pin to minimize the capstan effect. Calibrated masses were suspended from the monofilament line. This allowed for repeatable lateral contact force magnitudes and directions to be imparted on the nozzle.

We applied lateral forces along $F_{x}$ and $F_{y}$ with a range of $0.1-7 \mathrm{~N}$ to the nozzle tip. These measurements allowed for the skew correction parameters and lateral linear coefficients to be determined. Skew correction parameters were determined fitting lines to the measured force representations using least squares. The angle between this line and the respective axis was measured. Angle parameters $a$ and $b$ were then inputted into the sensor model (Appendix).

We used the same data to find linear scaling coefficients $L_{x}$ and $L_{y}$. Skewed, rotated, unscaled lateral force representations $X_{\text {skew }}$ and $Y_{\text {skew }}$ were plotted against the applied lateral contact force in 


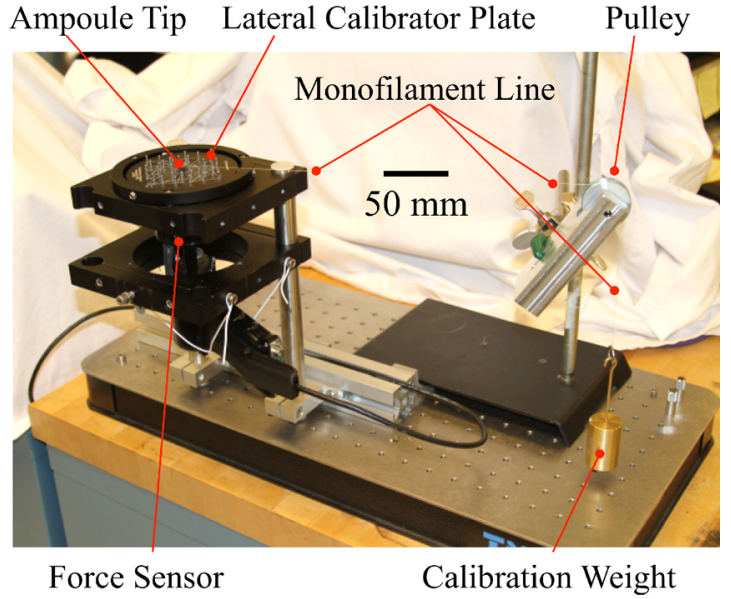

Fig. 10 The lateral contact force calibration jig being used to calibrate lateral contact force magnitude and direction. The monofilament line extends perpendicularly from the vertically mounted nozzle so that no normal contact force was exerted on the nozzle.

the pure $x$ or $y$ direction and a line was fit using least squares. The slopes indicated the lateral scaling coefficient. After skew and calibration were implemented, a range of lateral force directions were applied at magnitudes of $1.98 \mathrm{~N}$ and $4.92 \mathrm{~N}$ and the results are shown in Fig. 11. The measured lateral force magnitude mean absolute error from the applied magnitude is $101 \mathrm{mN}$ with a maximum absolute error of $357 \mathrm{mN}$ and no systematic trends. The measured lateral force angle mean absolute error from the applied angle is $1.60 \mathrm{deg}$ with a maximum error of $-0.79 \mathrm{deg}$ and a minimum error of -3.21 deg indicating a negligible systematic trend. The standard deviation of each individual $F_{x}$ and $F_{y}$ measurement is $54 \mathrm{mN}$ and $56 \mathrm{mN}$, respectively.

We conducted finite element analysis (FEA) on the sensor structure for both static loads and dynamic resonances (SolidWorks). The static performance of the sensor was evaluated under

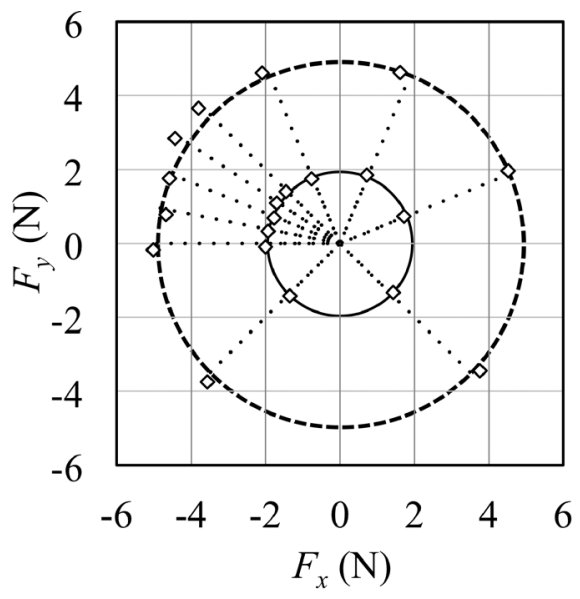

$\diamond$ Lateral Force Sensor Measurement

- $1.98 \mathrm{~N}$ Expected Magnitude

--- $4.92 \mathrm{~N}$ Expected Magnitude

... Expected Angle

Fig. 11 Experimentally measured lateral forces fall very close to the expected location at the intersection between the radial lines (representing applied angles) and circles (representing applied magnitudes) a static axial load of $300 \mathrm{~N}$, as explained in Sec. 2.1. The axial displacement result of $24.19 \mu \mathrm{m}$ (Fig. 12) would induce a maximum expected volume error of $0.23 \mu \mathrm{L}$ during an injection (given the internal diameter of the Injex U-100 ampoule of $3.5 \mathrm{~mm}$ ). Presuming a linear relationship between the force and displacement, we would expect a maximum volume error of $0.15 \mu \mathrm{L}$ at the maximum force output of the pre-existing actuator [1]. The displacements caused by the $100 \%$ and $150 \%$ loading conditions are less than the maximum allowable axial displacement of $25 \mu \mathrm{m}$ (as specified in Sec. 2.1) and was therefore deemed acceptable. Since the yield stress is not exceeded [19], the deformation is elastic. Additionally, this 7075-T6 aluminum resists a large number of loading cycles (upwards of $10^{6}[18]$ ) at the maximum stress simulated in FEA (Fig. 13). These characteristics meet the design constraint to repeatably withstand $300 \mathrm{~N}$. Dynamic resonances were calculated in FEA and all fall above $7 \mathrm{kHz}$ [16], which is above the requirement of $5 \mathrm{kHz}$ specified in Sec. 2.1.

While FEA had indicated minimal volume error induced by the sensor during an injection, experimentally confirming a repeatable volume delivery was critical to clear the sensor for biological testing. The experimental validation setup and procedure were identical to previous volume validation experiments [1]. Jet velocity was set at $150 \mathrm{~m} / \mathrm{s}$ and target ejected volumes were set at either $50 \mu \mathrm{L}$ or $100 \mu \mathrm{L}$. Ejected volume is the sum of volume delivered and volume remaining on the tissue surface (wet injection). The original jet injector without the force sensor achieved average ejected volumes of $48.3 \pm 1.8 \mu \mathrm{L}$ and $100.7 \pm 0.4 \mu \mathrm{L}$ at target volumes of $50 \mu \mathrm{L}$ and $100 \mu \mathrm{L}$, respectively, whereas the jet injector with the force sensor installed achieved average ejected volumes of $50.0 \pm 0.5 \mu \mathrm{L}$ and $100.6 \pm 0.2 \mu \mathrm{L}$ at target volumes of $50 \mu \mathrm{L}$ and $100 \mu \mathrm{L}$, respectively (Fig. 14). These results are reported as the mean \pm one standard deviation.

We used a two-tailed $t$-test to compare the accuracy between jet injectors with and without the force sensor. Accuracy was defined as the mean difference between observed ejected volume and target ejection volume. At a target volume of $50 \mu \mathrm{L}$, the $t$-statistic was 5.2 (two-tailed $p<0.01$ ) so we reject the null hypothesis that

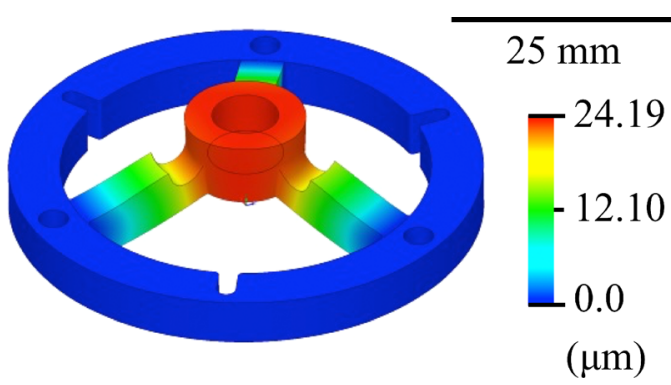

Fig. 12 FEA displacement results due to $300 \mathrm{~N}$ static axial load. Deformation is exaggerated for visualization purposes $(200 \times)$.

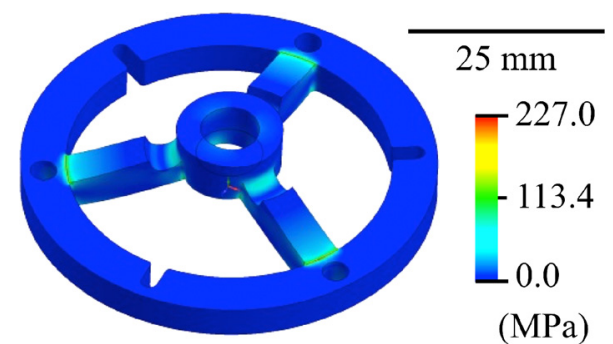

Fig. 13 FEA von Mises stress results due to $300 \mathrm{~N}$ static axial load. Plastic deformation would not occur as the yield stress is $503 \mathrm{MPa}$ for 7075-T6 aluminum alloy [19]. Deformation is exaggerated for visualization purposes $(200 \times)$. 
$50 \mu \mathrm{L}$ Ejection Validation

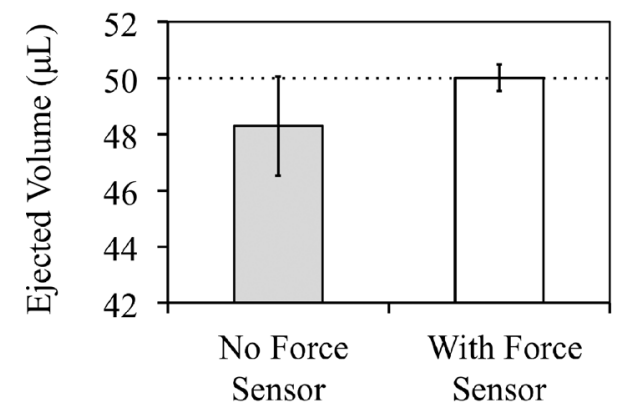

$100 \mu \mathrm{L}$ Ejection Validation

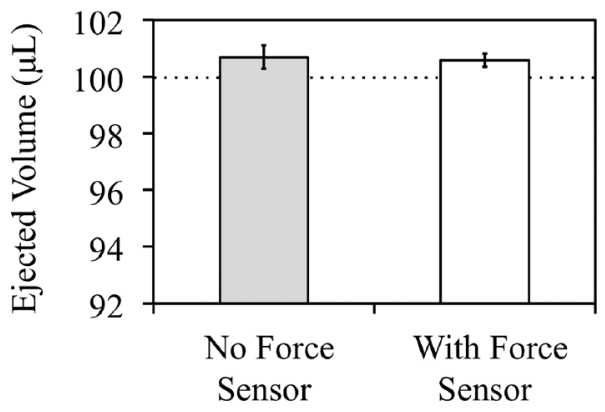

Fig. 14 These plots compare the results from the ejection validation experiments conducted with the pre-existing jet injector [1] for various volumes and jet velocities. The black dashed line shows the target volume. Shown in gray boxes are results with the jet injector without force sensor. The white boxes show the volume ejection performance with the force sensor. For all tests, $n=30$. The error bars indicate one standard deviation above and below the mean.

the accuracy of the jet injector with and without the force sensor are equal, at a significance level of $1 \%$. At a target volume of $100 \mu \mathrm{L}$, the $t$-statistic was -1.8 (two-tailed $p=0.08$ ) so we fail to reject the null hypothesis that the accuracy of the jet injector with and without the force sensor are equal, at a significance level of $1 \%$. Thus, we conclude that including the force sensor does not negatively affect the accuracy of volume ejected from the jet injector, as its presence significantly improved the accuracy of the jet injector at $50 \mu \mathrm{L}$ but had no significant effect on the accuracy of the jet injector at $100 \mu \mathrm{L}$.

To compare the precision of the jet injector with and without the force sensor, we conducted a Levene's test [20]. We define precision as the reciprocal of the variance of the observed ejected volume. At a target volume of $50 \mu \mathrm{L}$, the Levene's robust test statistic was $35.9(p<0.01)$ so we can reject the null hypothesis that the variances are equal between the jet injector with and without the force sensor, at a significance level of $1 \%$. At a target volume of $100 \mu \mathrm{L}$, the Levene's robust test statistic was $0.1(p=0.74)$ so we fail to reject the null hypothesis that the variances are equal between the jet injector with and without the force sensor, at a significance level of $1 \%$. Similar to our conclusion above for the accuracy of the device, we conclude that the inclusion of the force sensor does not negatively affect the precision of volume ejected from the jet injector, as it significantly improved the precision of the jet injector at $50 \mu \mathrm{L}$ but had no significant effect on the precision of the jet injector at $100 \mu \mathrm{L}$.

\section{Biological Testing}

3.1 Procedure. With the sensor fully calibrated and ejection performance validated, we administered injections into pieces of abdominal porcine tissue, harvested from animals 6 months old or less, which included the epidermis, dermis, subcutaneous fat, and top muscle layers. The tissue was frozen at $-80^{\circ} \mathrm{C}$ immediately after harvesting. This tissue block was trimmed to an approximate width of $80 \mathrm{~mm}$, length of $62 \mathrm{~mm}$, and height of $25 \mathrm{~mm}$. We placed the block atop a piece of absorbent paper towel on a glass plate and allowed it to thaw prior to injections. This was the first and only time the tissue was allowed to thaw. After thawing was complete (2-3h at room temperature), we moved the tissue to a clear polymer tray and performed injections. As with the ejection validation testing, we fired three different ampoules ( $a, b, c)$, and we exchanged each after every injection sequentially $(\mathrm{a}, \mathrm{b}, \mathrm{c}, \mathrm{a}, \mathrm{b}, \mathrm{c} \ldots)$ to control for ampoule and piston variability and wear [21]. We presented the user with an interface that showed normal and lateral force measurements and the user's deviation from a specified normal contact force and zero lateral force. We varied the normal contact force specification randomly between low $(0-3.9 \mathrm{~N})$ and high (4-8N) between the injection sites to control for local tissue variation. The user was able to achieve a mean normal force error of $0.67 \mathrm{~N}$ with a standard deviation of $0.68 \mathrm{~N}$ for these tests. We note that this is outside the range originally specified in Sec. 2.1. However, having a smaller range as a constraint simply sets the performance specification higher than was perhaps necessary. Lateral contact forces were simultaneously minimized. The user was able to achieve a mean lateral force magnitude of $0.68 \mathrm{~N}$ with a standard deviation of $0.40 \mathrm{~N}$ for these tests.

For these injections, $v_{\text {jet }}=200 \mathrm{~m} / \mathrm{s}, t_{\text {jet }}=10 \mathrm{~ms}, v_{\text {follow }}=50 \mathrm{~ms}$, and $V=100 \mu \mathrm{L}$ [1]. Note that $v_{\text {follow }}$ is significantly less than $v_{\text {jet }}$ and $v_{\text {follow }}$ is maintained for the time necessary to reach the desired $V$ given the diameter of the ampoule. We completed 12 total injections per tissue block. We injected water mixed with tissue-marking dye on a grid with a spacing of approximately $25 \mathrm{~mm}$. We dabbed the tissue surface with a towelette before injection, and any liquid remaining on the surface after injection was carefully absorbed after each injection. An image of the tissue block after injections from a top view is shown in Fig. 15. We determined the fluid volume remaining on the surface using the mass method [1,16,21]. To determine the volume delivered (as reported in Fig. 16), we subtracted the volume remaining on the surface from the target ejected volume.

3.2 Results. We compared the volume delivered between low normal contact force $(0-3.9 \mathrm{~N})$ and high normal contact force (4-8 N). $100 \mu \mathrm{L}$ injections occurring at low normal contact force had an average volume delivered of $68.9 \mu \mathrm{L}(12.7 \mu \mathrm{L}$ standard deviation, $44.8 \mu \mathrm{L}$ tolerance interval). As mentioned in Sec. 1, the tolerance interval indicates the absolute error within which $93 \%$ of all doses will be delivered. In contrast to injections at this low normal contact force range, injections occurring at high normal

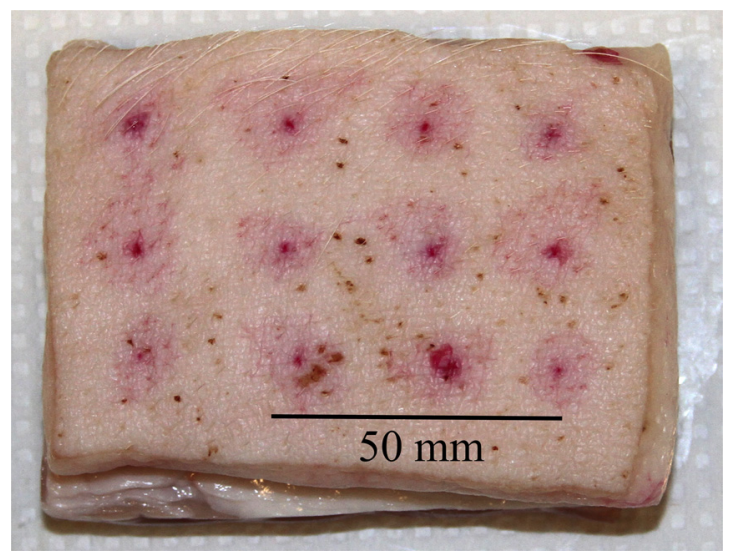

Fig. 15 The top of the porcine tissue block after being injected with water mixed with tissue-marking dye at an ejection volume of $100 \mu \mathrm{L}$. The injection locations are visible as red dots (resulting from the injectate penetrating the tissue) in a regular grid pattern. 


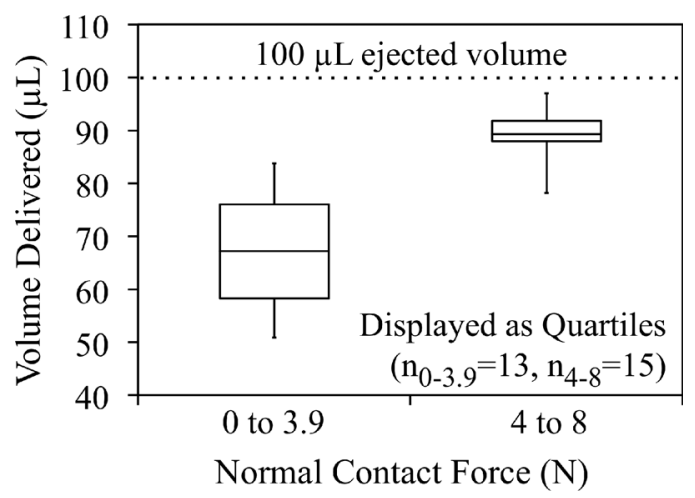

Fig. 16 The relationship between volume delivered and normal contact force for $100 \mu \mathrm{L}$ injections into porcine tissue using a $v_{\text {jet }}$ of $200 \mathrm{~m} / \mathrm{s}, t_{\text {jet }}$ of $10 \mathrm{~ms}$, and $v_{\text {follow }}$ of $50 \mathrm{~m} / \mathrm{s}$ at a low and high normal contact force ranges

contact forces showed increased volume delivery with smaller standard deviation at $89.1 \mu \mathrm{L}(5.1 \mu \mathrm{L}$ standard deviation, $17.3 \mu \mathrm{L}$ tolerance interval). Using a two-tailed $t$-test, we compare the accuracy of volume delivered under low normal contact force with that made with high normal contact force. The $t$-statistic was 5.4 (two-tailed $p<0.01$ ) so we reject the null hypothesis that the mean volume delivered between low and high normal contact force are equal at a significance level of $1 \%$. A Levene's test was conducted to compare the precision of volume delivered between injections with low normal contact force and those with high contact force. The Levene's robust test statistic was $15.6(p<0.01)$ so we reject the null hypothesis that the variances of volume delivered between low and high normal contract force are equivalent at a significance level of $1 \%$. The experimental results confirm that the magnitude of normal contact force affects both the accuracy and precision of volume delivered using medical jet injection. These results are shown in Fig. 16.

Finally, we revisit the ISO accuracy specifications for needlebased injection systems for medical use [6] and compare these requirements to the accuracy of the volume delivered by the jet injection system at low and high normal contact force ranges detailed above. For a target volume of $100 \mu \mathrm{L}$, the standard specifies an absolute error of $10 \mu \mathrm{L}$. While the tolerance interval for volume delivered by the jet injector at both low and high normal contact force ranges do not meet this specification, our results indicate that the accuracy and precision of volume delivered from medical jet injectors can be significantly improved by increasing the normal contact force from a low range (0 to $3.9 \mathrm{~N})$ to a high range $(4$ to $8 \mathrm{~N})$.

\section{Conclusions}

We have presented a design for a novel, multi-axis electronic force sensor optimized for medical jet injection. Through calibration and validation with methods described, the sensor meets all quantitative and qualitative constraints originally specified. While not yet as reliable as commercial needle-based injectors [7], our results indicate that the accuracy and precision of volume delivered from medical jet injectors can be significantly improved by controlling for normal contact force. This result is important given a need to reduce the incidence rate and severity of wet injections (fluid drug remaining on the tissue surface) in medical jet injection. The sensor (with feedback interface) is readily capable of reliably guiding users to apply a normal force level of $4-8 \mathrm{~N}$ (the desirable range determined in this study) while also minimizing lateral forces.

In speculating on the reasons why higher levels of normal contact force improve volume delivery, we can identify two possible mechanisms. First, changing the normal contact force could vary the quality of the circumferential seal formed around the nozzle.
We define seal quality as the maximum pressure that the nozzle-tissue interface can contain. The effect of normal contact force on seal quality is most likely dependent on the design of the nozzle tip. Second, penetrability of the target could vary with normal contact force as it does with other parameters indicated in the literature [11,22]. We define penetrability as the inverse of the fluid stagnation pressure required for skin penetration to the required depth. Further studies could investigate on whether elevated normal contact force improves seal quality and/or penetrability.

A number of other future studies may also be pursued. Probing the relationship between volume delivered and contact force under conditions different from those presented in this study would be of interest. These efforts may indicate a need to specify different normal contact force for different injection conditions, which is readily possible with this electronic force sensor. These conditions could include the mechanical properties of tissue, injectate viscosity, injectate volume, nozzle diameter, nozzle shape, and jet speed among others. While lateral forces were minimized in this study, a future study could determine the effect of lateral forces on volume delivered. Larger injection studies carried out in living tissue would be beneficial to confirm and refine the findings presented in this study. Finally, one design change that would be useful to implement in future force sensors is the ability to monitor orientation. In this way, systematic errors caused by the weight of the ampoule could be removed.

\section{Acknowledgment}

The authors would like to thank N. C. Hogan for preparing the tissues used in this study, M. T. Nawrot for the unmodified jet injector experimental volume validation data presented in Fig. 14, and N. Nakajima for assistance with the statistical analysis. The authors would also like to thank N. Nakajima and G. J. Wang for proofreading this manuscript.

\section{Nomenclature}

$a=X$-axis skew correction angle

$b=Y$-axis skew correction angle

$C_{n}=$ calibration coefficient for flexural beam $n$

$F_{n}=$ force supported by flexural beam $n$

$F_{x}=$ lateral contact force applied to the nozzle tip in the $x$ direction

$F_{y}=$ lateral contact force applied to the nozzle tip in the $y$ direction

$F_{\text {applied }}=$ force applied to the jet injector by the user that is normal to the tissue surface

$F_{\text {normal }}=$ normal contact force applied to the nozzle tip

$L_{x}=$ lateral calibration coefficient for $X$ skew

$L_{y}=$ lateral calibration coefficient for $Y$ skew

$M_{\text {applied }}=$ torque applied to the jet injector by the user

$t_{\text {jet }}=$ duration of time for which $v_{\text {jet }}$ is sustained

$V=$ target ejected volume

$v_{\text {follow }}=$ jet velocity during the follow-through phase of delivery

$v_{\text {jet }}=$ jet velocity during the initial phase of delivery

$V_{n}=$ voltage measured from flexural beam $n$

$X=$ unscaled lateral force representation in the $x$ direction

$X_{\text {rot }}=$ rotated, unscaled lateral force representation in the $x$ direction

$X_{\text {skew }}=$ skewed, rotated, unscaled lateral force representation in the $x$ direction

$Y=$ unscaled lateral force representation in the $y$ direction

$Y_{\text {rot }}=$ rotated, unscaled lateral force representation in the $y$ direction

$Y_{\text {skew }}=$ skewed, rotated, unscaled lateral force representation in the $y$ direction

$Z_{n}=$ offset voltage for flexural beam $n$

$\theta=$ rotational correction angle 


\section{Appendix: Rotation and Skew Correction}

Rotation correction, which rotates the measured coordinate system by angle parameter $\theta$ (determined by the angle at which the sensor is mounted), aligns the instrument's axes with the user's axes. This transformation is represented in the schematic Fig. 17 and as the rotational matrix equation in the following equation:

$$
\left[\begin{array}{c}
X_{\text {rot }} \\
Y_{\text {rot }}
\end{array}\right]=\left[\begin{array}{cc}
\cos (\theta) & \sin (\theta) \\
-\sin (\theta) & \cos (\theta)
\end{array}\right]\left[\begin{array}{c}
X \\
Y
\end{array}\right]
$$

Skew correction adjusts the angle of the lateral forces to ensure that the $Y_{\text {rot }}$ and $X_{\text {rot }}$ measurements in the lateral plane are orthogonal. It utilizes angle parameters $a$ and $b$, which are determined

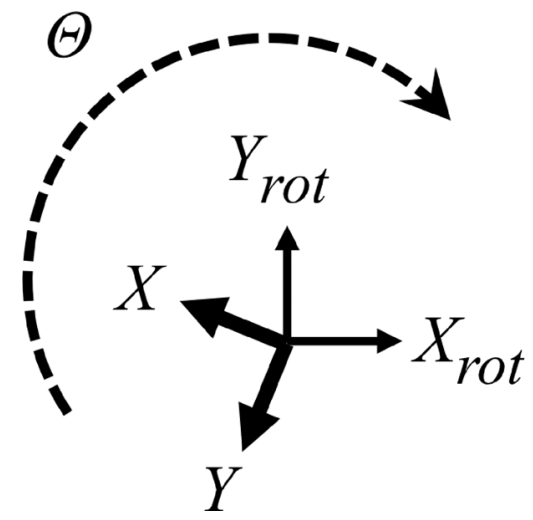

Fig. 17 Rotation correction schematic showing how the sensor's axes are aligned with the user's axes using Eq. (A1). Presuming the user's axes are oriented like a traditional Cartesian coordinate system for the reader, the sensor is mounted at a rotation of $-\theta$ due to geometric requirements of the jet injector. A rotation about the origin by angle $\theta$ is required to align the sensor's axes with the user's axes.

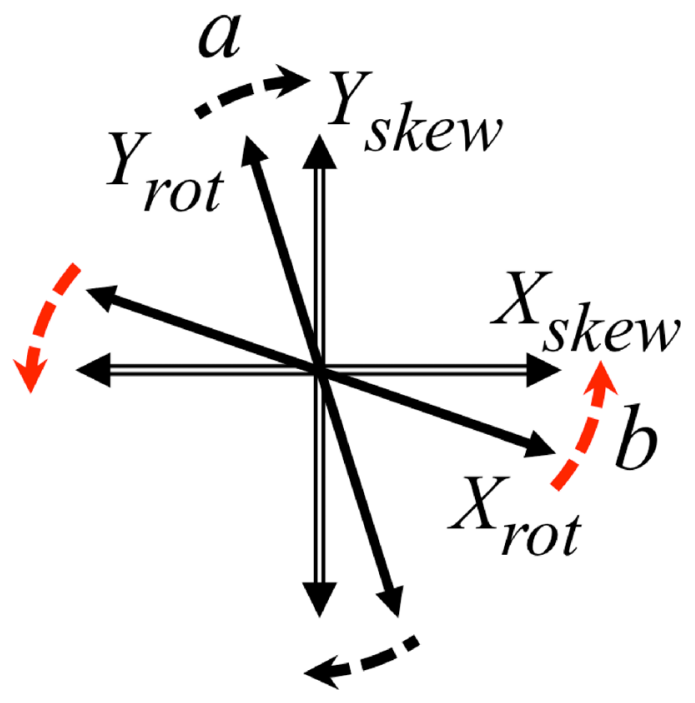

Fig. 18 Skew correction schematic indicating how the sensor's lateral axes are orthogonalized using Eq. (A2). The input axis system is skewed by angle parameters $a$ and $b$, which relate the original and desired $y$-and $x$-axes, respectively. experimentally, to correct the $Y_{\text {rot }}$ and $X_{\text {rot }}$ to $Y_{\text {skew }}$ and $X_{\text {skew }}$, respectively. This is represented in the schematic Fig. 18 and as the matrix equation in the following equation:

$$
\left[\begin{array}{c}
X_{\text {skew }} \\
Y_{\text {skew }}
\end{array}\right]=\left[\begin{array}{cc}
1 & -\tan (b) \\
-\tan (a) & 1+\tan (b) \tan (a)
\end{array}\right]\left[\begin{array}{c}
X_{\text {rot }} \\
Y_{\text {rot }}
\end{array}\right]
$$

\section{References}

[1] Taberner, A. J., Hogan, N. C., and Hunter, I. W., 2012, "Needle-Free Jet Injection Using Real-Time Controlled Linear Lorentz-Force Actuators," Med. Eng. Phys., 34(9), pp. 1228-1235.

[2] Hoffman, P. N., Abuknesha, R. A., Andrews, N. J., Samuel, D., and Lloyd, J. S., 2001, "A Model to Assess the Infection Potential of Jet Injectors Used in Mass Immunisation," Vaccine, 19(28-29), pp. 4020-4027.

[3] Engwerda, E. E. C., Tack, C. J., and de Galan, B. E., 2013, “Needle-Free Jet Injection of Rapid-Acting Insulin Improves Early Postprandial Glucose Control in Patients With Diabetes," Diabetes Care, 36(11), pp. 3436-3441.

[4] Ferayorni, A., Yniguez, R., Bryson, M., and Bulloch, B., 2012, "Needle-Free Jet Injection of Lidocaine for Local Anesthesia During Lumbar Puncture: A Randomized Controlled Trial," Pediatr. Emerg. Care, 28(7), pp. 687-690.

[5] Hogan, N. C., Taberner, A. J., Jones, L. A., and Hunter, I. W., 2015, "NeedleFree Delivery of Macromolecules Through the Skin Using Controllable Jet Injectors," Expert Opin. Drug Delivery, 12(10), pp. 1637-1648.

[6] ISO, 2014, "Needle-Based Injection Systems for Medical Use-Requirements and Test Methods-Part 1: Needle-Based Injection Systems," International Organization for Standardization, Geneva, Switzerland, Standard No. 11608$1: 2014(\mathrm{E})$

[7] Krzywon, M., van der Burg, T., Fuhr, U., Schubert-Zsilavecz, M., and AbdelTawab, M., 2012, "Study on the Dosing Accuracy of Commonly Used Disposable Insulin Pens," Diabetes Technol. Ther., 14(9), pp. 804-809.

[8] Schramm, J., and Mitragotri, S., 2002, "Transdermal Drug Delivery by Jet Injectors: Energetics of Jet Formation and Penetration," Pharm. Res., 19(11), pp. 1673-1679.

[9] Schramm-Baxter, J., Katrencik, J., and Mitragotri, S., 2004, "Jet Injection Into Polyacrylamide Gels: Investigation of Jet Injection Mechanics," J. Biomech., 37(8), pp. 1181-1188.

[10] Wendell, D. M., Hemond, B. D., Hogan, N. C., Taberner, A. J., and Hunter, I. W., 2006, "The Effect of Jet Parameters on Jet Injection," International Conference of the IEEE Engineering in Medicine and Biology Society (IEMBS), New York, Aug. 30-Sept. 3, pp. 5005-5008.

[11] Shergold, O. A., Fleck, N. A., and King, T. S., 2006, "The Penetration of a Soft Solid by a Liquid Jet, With Application to the Administration of a Needle-Free Injection," J. Biomech., 39(14), pp. 2593-2602.

[12] Rajaratnam, N., Rizvi, S. A. H., Steffler, P. M., and Smy, P. R., 1994, "An Experimental Study of Very High Velocity Circular Water Jets in Air," J. Hydraul. Res., 32(3), pp. 461-470.

[13] Park, G., Modak, A., Hogan, N. C., and Hunter, I. W., 2015, "The Effect of Jet Shape on Jet Injection," 37th Annual International Conference of the IEEE Engineering in Medicine and Biology Society (EMBC), Milan, Italy, Aug. 25-29, pp. 7350-7353.

[14] Hemond, B. D., Taberner, A. J., Hogan, N. C., Crane, B., and Hunter, I. W., 2011, "Development and Performance of a Controllable Autoloading NeedleFree Jet Injector," ASME J. Med. Devices, 5(1), p. 015001.

[15] Stachowiak, J. C., von Muhlen, M. G., Li, T. H., Jalilian, L., Parekh, S. H., and Fletcher, D. A., 2007, "Piezoelectric Control of Needle-Free Transdermal Drug Delivery," J. Controlled Release, 124(1-2), pp. 88-97.

[16] Demas, N. P., 2015, “A Contact Force Sensor for Medical Jet Injection,” Master's thesis, Massachusetts Institute of Technology, Cambridge, MA.

[17] Demas, N. P., Hunter, I. W., Hemond, B. D., and Nawrot, M. T., 2016 "Multi-Directional Low-Displacement Force Sensor," U.S. Patent No. US9724473B2.

[18] Grandt, A., 2003, Fundamentals of Structural Integrity: Damage Tolerant Design and Nondestructive Evaluation, Wiley, Hoboken, NJ.

[19] Oberg, E., Jones, F., Horton, H., and Ryffel, H., 2008, Machinery's Handbook, 28th ed., Industrial Press, New York.

[20] Gastwirth, J., Gel, Y., and Miao, W., 2009, "The Impact of Levene's Test of Equality of Variances on Statistical Theory and Practice," Stat. Sci., 24(3), pp. 343-360.

[21] Nawrot, M. T., 2014, "Design of a Robust, Intuitive Piston Interface for a Needle Free Injection System," Master's thesis, Massachusetts Institute of Technology, Cambridge, MA.

[22] Shergold, O. A., and Fleck, N. A., 2005, "Experimental Investigation Into the Deep Penetration of Soft Solids by Sharp and Blunt Punches, With Application to the Piercing of Skin," ASME J. Biomech. Eng., 127(5), pp. 838-848. 